\title{
Análise Comportamental por Meio de Características com os Classificadores SVM e MLP Para Detecção de Traços Autísticos em Adultos
}

\author{
Roney Nogueira de Sousa ${ }^{1}$, Rhyan Ximenes de Brito ${ }^{1}$, \\ Janaide Nogueira de Sousa Ximenes ${ }^{2}$ \\ ${ }^{1}$ Instituto Federal de Educação, Ciência e Tecnologia do Ceará (IFCE) \\ Av. Tabelião Luiz Nogueira de Lima S/N - Tianguá - CE - Brazil \\ ${ }^{2}$ Faculdade IEducare (FIED) - Rua Conselheiro João Lourenço, \\ 406 - CEP 62320-000 - Tianguá - CE - Brasil \\ \{nogueiraroney453, rxbrito, nogueirajanaide\}@gmail.com
}

\begin{abstract}
Autistic Spectrum Disorder (ASD) intrigues researchers worldwide. In this sense, this work addresses a study on the use of Artificial Intelligence (AI) as an auxiliary tool for ASD screening in adults. This work aimed to develop an auxiliary system based on the SVM and MLP classifiers as a tool for detecting autistic traits in adults. The methodology was implemented based on 610 samples of individuals with/without TEA from a public database, through WEKA software using SVM and MLP, cross validation $k$-fold, $z$-score and the SMOTE technique. The results showed for the SVM accuracy an average rate of 99.48\% while the MLP was $95.42 \%$.
\end{abstract}

Resumo. O transtorno do especto autista (TEA) intriga pesquisadores no mundo inteiro. Nesse sentido esse trabalho aborda um estudo sobre o uso da Inteligência Artificial (IA) como ferramenta auxiliar para rastreio do TEA em adultos. Este trabalho teve como objetivo desenvolver um sistema auxiliar com base nos classificadores SVM e MLP como ferramenta de detecção de traços autísticos em adultos. A metodologia foi implementada com base em 610 amostras de indivíduos com/sem o TEA de um banco de dados público, através do software WEKA utilizando o SVM e a MLP, validação cruzada $k$-fold, $z$-score e a técnica SMOTE. Os resultados mostraram para a acurácia do SVM uma taxa média de 99,48\% enquanto que para a MLP foi de 95,42\%.

\section{Introdução}

O transtorno do espectro autista (TEA) caracteriza-se por apresentar prejuízos no desenvolvimento de habilidades sociais persistentes na comunicação, interação social e linguagem limitando-os ou prejudicando-os diáriamente [Onzi and de Figueiredo Gomes 2015]. Inicia-se normalmente antes dos três anos de idade, ainda não se compreendendo totalmente as causas que levam ao seu desenvolvimento [Souza 2016].

Nessa perspectiva sabe-se que o uso de técnicas de Inteligência Computacional Aplicada (ICA) tem se destacado como mecanismo facilitador na busca por respostas frente aos mais diversos tipos de problemas nas mais diversas áreas do conhecimento, 
ganhando destaque na saúde, educação, engenharia entre outras. Com base nessa perspectiva realizou-se um estudo com o classificador Support Vector Machine (SVM) e Multilayer Perceptron (MLP) visando descobrir através da classificação traços autísticos em adultos.

O uso da SVM e da MLP se deu por conta que os resultados da aplicação dessas técnicas são comparáveis e muitas vezes superiores aos obtidos por outros algoritmos de aprendizado de máquina [Lorena and de Carvalho 2007].

Esse trabalho tem como principal objetivo realizar um estudo utilizando os classificadores SVM e MLP que se encontram disponíveis no software Waikato Environment for Knowledge Analysis (WEKA), como mecanismo de auxílio no processo de identificação de traços autísticos em adultos. O estudo foi realizado a partir do aprofundamento bibliográfico acerca do assunto e no emprego dos classificadores SVM e MLP com base em um dataset público composto por amostras de adultos com/sem o TEA, intitulado "Autism Screening Adult Data Set" obtido através do link https://archive.ics.uci.edu/ml/datasets/Autism+Screening+Adult, além de outras técnicas como a normalização.

Este trabalho está organizado em 6 seções: a Seção 2 apresenta os trabalhos relacionados; Seção 3 traz a fundamentação teórica sobre o TEA e os classificadores SVM e MLP; a Seção 4 apresenta a metodologia; Seção 5 trata dos resultados e discussões; e a Seção 6 expoe as considerações finais e trabalhos futuros.

\section{Trabalhos Relacionados}

Esta seção apresenta uma revisão bibliográfica de trabalhos que abordam a utilização de técnicas de Inteligência Artificial como ferramentas de auxílio ou rastreio para o diagnóstico de problemas na área de saúde.

[Zavaleta et al. 2012] apresentou uma ferramenta modular baseada em técnicas de Inteligência Computacional (IC) que visava apoiar especialistas na detecção, avaliação e intervenção no diagnóstico da dislexia. O estudo foi realizado com base em redes neurais não-supervisionadas para detectar e classificar os padrões da dislexia, utilizando amostras de 52 estudantes entre 09 e 18 anos.

[Pinheiro 2018] implementou um sistema de classificação que dada uma imagem facial de uma criança, conseguisse discriminar entre os dois grupos, com/sem TEA, auxiliando assim no diagnóstico. As imagens foram processadas, testadas e comparadas a diferentes métodos de redução de dimensionalidade e classificação e como resultado obteve uma acurácia de $80 \%$ de acertos com o classificador Random Forest.

[Araujo et al. 2019] desenvolveu um sistema baseado em regras fuzzy para um pré-diagnóstico, objetivando auxiliar psiquiatras no diagnóstico da esquizofrenia. Teve como base pesquisas bibliográficas e simulações com pacientes fictícios, utilizando como variáveis de entrada alguns sintomas tais como: delírios, alucinações, entre outras. Os resultados foram promissores mostrando-se bem próximos dos observados por profissionais da saúde.

[de Brito et al. 2019] realizaram um estudo com a implementação e análise das redes Multilayer Perceptron (MLP) e Radial Basis Function Neural Network (RBF), objetivando comparar resultados baseados no treinamento, teste e classificação de crianças 
com/sem autismo. Os resultados foram analisados considerando as características e os comportamentos diferentes das redes implementadas, obtendo-se uma medida da qualidade atingida.

\section{Fundamentação Teórica}

Esta seção traz um estudo sobre o transtorno do espectro autista abordando também os classificadores Support Vector Machine e Multilayer Perceptron (MLP) com suas particularidades.

\subsection{O Transtorno do Espectro Autista em Adultos}

O psiquiatra suíço Paul Eugen Bleuler utilizou o termo autismo pela primeira vez em 1911 para descrever um sintoma de esquizofrenia definido como desligamento da realidade combinado com a predominância relativa ou absoluta da vida interior [Caminha et al. 2016]. No início dos anos 1940, com as obras de Kanner (publicada em 1943) e Asperger (publicada em 1944) surgiram as primeiras descrições clínicas do autismo [Martins 2017].

Para [Carvalho et al. 2016] o autismo é um distúrbio do desenvolvimento, caracterizado por atrasos graves e específicos, com disfunções na comunicação e no desenvolvimento social e cognitivo. Para [Borges and Shinohara 2007] a síndrome pode ser definida também como perda de contato com a realidade, causada pela impossibilidade ou grande dificuldade na comunicação interpessoal. Contudo [Bentes et al. 2016] afirma que está intimamente relacionado a dificuldades de socialização, agressividade, interesse restrito por assuntos ou podem deter habilidade verbal e cognitiva desenvolvida, estas características variam de acordo com o quadro .

Sabe-se que o autismo é considerado atualmente como uma disfunção cerebral orgânica graças a várias evidências. Entre elas, um retardo mental está associado ao autismo em $70 \%$ dos casos e convulsões em 33\% dos casos [Zilbovicius et al. 2006]. A presença desses traços variam em quantidade e intensidade. Portanto, é possível encontrar pessoas com autismo que apresentem sintomas severos e por isso necessitarem de maior acompanhamento, bem como pessoas que manifestam traços mais leves e consequentemente, menos prejuízos [Alves et al. 2019].

O diagnóstico é clínico e baseia-se em um desenvolvimento atípico ou deficiente da comunicação social e da interação social apresentando um repertório restrito de comportamentos, atividades e interesses [Caminha et al. 2016]. Para o diagnóstico do TEA utiliza-se como referência o apresentado no Manual de Diagnóstico e Estatístico de Transtornos Mentais $5^{\mathrm{a}}$ edição (DSM-5).

O DSM-5 apresenta uma nova classificação abrangendo quatro condições assim assinaladas: déficit em comunicação; em interação social; em padrão de comportamento; e atividades e interesses restritivos e repetitivos [Freire Monteiro et al. 2018].

É importante destacar que o TEA e caracterizado por um conjunto muito heterogêneo de alterações comportamentais com início precoce, curso crônico e impacto variável em áreas múltiplas do desenvolvimento [da Silva Talarico et al. 2019]. 


\subsection{Support Vector Machine (SVM)}

A SVM constitui uma técnica de aprendizado que vem recebendo crescente atenção da comunidade de Aprendizado de Máquina [Lorena and de Carvalho 2007]. O SVM baseia-se nos princípios da minimização do risco estrutural, proveniente da teoria do aprendizado estatístico, a qual está baseada no fato de que o erro do algoritmo de aprendizagem junto aos dados de validação é limitado pelo erro de treinamento mais um termo que depende da dimensão Vapnik e Chervonenkis [Semolini et al. 2002].

Os fundamentos de SVM são provenientes da Teoria de Aprendizagem Estatística desenvolvida inicialmente pelo pesquisador russo Vladmir Vapnik e colaboradores. Vapnik idealizou o princípio indutivo de Minimização do Risco Estrutural. Este princípio busca minimizar o erro do conjunto de treinamento, juntamente com o erro do conjunto de teste [Santos et al. 2002]. Vale destacar que as SVMs foram primeiramente desenvolvidas para resolverem problemas de classificação, estendendo-se em seguida para problemas de regressão [Santos 2019]. A Figura 1 nos mostra o funcionamento da SVM.

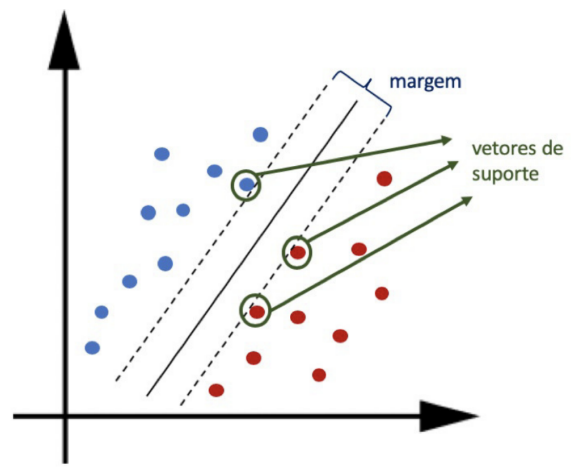

Figura 1. Funcionamento Classificador SVM

As funções usadas para projetar os dados do espaço de entrada para o espaço de alta dimensão são chamadas de kernels. Diferentes kernels têm sido propostos na literatura, são eles: lineares, polinomiais, gaussianas (mais comumente chamadas de RBF) e sigmóides. Diferentes definições da função Kernel e seus respectivos parâmetros provocam alterações nos resultados fornecidos por uma SVM [Souza et al. 2009]. A Tabela 1 mostras algumas expressões utilizadas em cada Kernel.

Tabela 1. Exemplos de Kernel e Funções

\begin{tabular}{l|c}
\hline Kernel & Função \\
\hline linear & $\mathrm{K}\left(\mathrm{x}^{\prime}, \mathrm{x}\right)=\mathrm{x} . \mathrm{x}^{\prime}$ \\
\hline polinomial com grau y & $\mathrm{K}\left(\mathrm{x}^{\prime}, \mathrm{x}\right)=\left(\gamma \cdot x^{\prime} \cdot x+y\right)^{d}$ \\
\hline RBF & $\mathrm{K}\left(\mathrm{x}^{\prime}, \mathrm{x}\right)=\exp \left(-\gamma \cdot\left\|x-x^{\prime}\right\|^{d}\right)$ \\
\hline Sigmóide & $\mathrm{K}\left(\mathrm{x}^{\prime}, \mathrm{x}\right)=\tanh \left(\gamma\left(x^{\prime} \cdot x\right)+y\right)$ \\
\hline
\end{tabular}

\subsection{Multilayer Perceptron (MLP)}

A rede neural MLP é uma rede perceptron do tipo feedforward que possui em sua estrutura um conjunto de unidades que formam a camada de entrada, uma ou mais camadas intermediarias ocultas de neurônios e uma camada de saída [Haykin et al. 2009]. 
A MLP é utiliza o treinado do tipo supervisionado em duas fases: (i) representa a propagação do sinal que parte da entrada e segue até saída; (ii) consiste na retro propagação do erro que se baseia na comparação da resposta obtida pela rede e a resposta desejada, utilizada no caminho reverso para atualizar os pesos da rede [Alves et al. 2016].

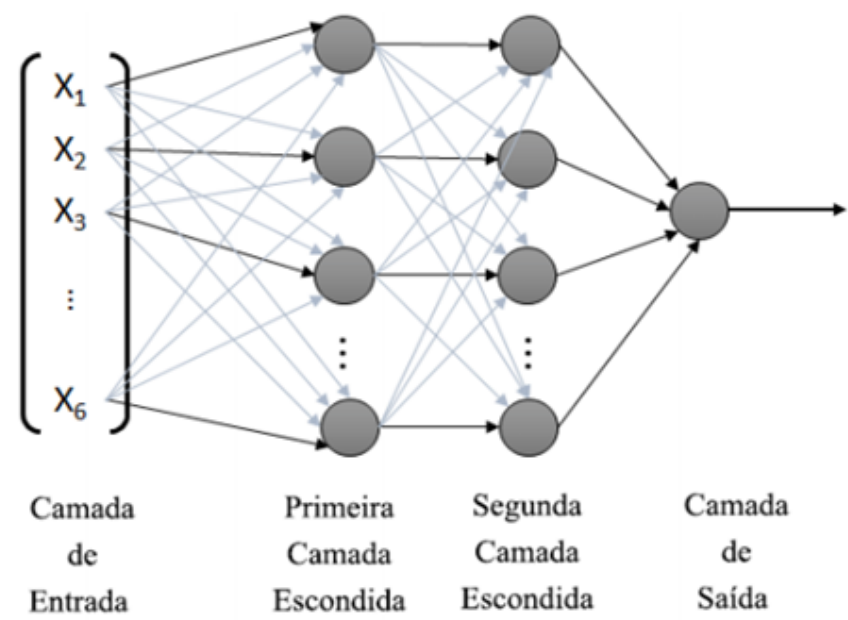

Figura 2. Arquitetura da Rede Neural MLP

A Figura 2 mostra a arquitetura da rede neural artificial MLP com uma camada de entrada, duas camadas ocultas ou intermediárias e uma camada de saída.

\section{Metodologia}

A metodologia desse trabalho foi desenvolvida em 2 fases: (i) base de dados e descrição da ferramenta utilizada; e (ii) treinamento e teste com a base de dados.

\subsection{Base de Dados e Descrição da Ferramenta Utilizada}

Utilizou-se uma base de dados pública intitulada Autism Screening Adult Data Set obtido através do link: https://archive.ics.uci.edu/ml/datasets/Autism+Screening+Adult, composta por 704 amostras e 21 atributos, dividida em 208 amostras de adultos não autista, representando cerca de $29,5 \%$ do total de amostras e 496 amostras de adultos autistas equivalente a $70,5 \%$.

\subsection{Treinamento e Teste com a Base de Dados}

Para o treinamento e teste utilizou-se os classificadores SVM e MLP. Para o SVM foram testados os kernels, RBF, sigmóide e linear com diferentes constantes de relaxamento. As melhores taxas de acurácias foram adquiridas com o kernel polinomial e constante de relaxamento de valor 1. Utilizou-se o algoritmo libSVM pertencente ao pacote de funções do software WEKA. Para a MLP testou vários hiperparâmetros, os melhores resultados foram observados com 2 camadas ocultas com 20 neurônios cada, função de ativação tangente hiperbólica, batch size de 0,5, learning rate de 0,2 com 200 epochs. Para o treinamento e teste foi utilizado o método de validação cruzada $k$-fold, com o k=10 folds, base de dados normalizada (z-score) e aplicação da técnica SMOTE para a criação de amostras sintéticas relacionadas a base de dados acrescentando-se 706 novas amostras totalizando 1.410. Porém logo após a realização do pré-processamento observou-se um total de 1.210 amostras. 


\section{Resultados e Discussões}

Os resultados e discussões analisados tiveram como base os percentuais de acurácia, especificidade e falso negativo adquiridos nos treinamentos e testes realizados com a base de dados aplicando os classificadores SVM e MLP.

Tabela 2. Resultados dos Testes com o SVM

\begin{tabular}{llcc}
\hline Folds & Taxa de Acerto (\%) & Taxa de erro (\%) & Situação \\
\hline 1 & 99,38 & 0,62 & \\
2 & 99,52 & 0,48 & \\
3 & 99,52 & 0,48 & \\
4 & 99,49 & 0,51 & \\
5 & 99,57 & 0,43 & Melhor Caso \\
6 & 99,49 & 0,51 & \\
7 & 99,52 & 0,48 & \\
8 & 99,49 & 0,51 & \\
9 & 99,52 & 0,48 & \\
10 & 99,31 & 0,69 & Pior Caso \\
\hline- & 99,48 & 0,52 & Caso Médio \\
\hline
\end{tabular}

Conforme observado na Tabela 2, percebe-se que o melhor caso representado pelo fold 5 atingiu uma acurácia de $99,57 \%$ de acertos e $0,43 \%$ de erros e o pior caso representado pelo fold 10 atingiu $99,31 \%$ de acertos e $0,69 \%$ de erros. A taxa média de acertos foi de $99,48 \%$ de acertos e $0,52 \%$ de erros. Percebeu-se também uma especificidade de $98,41 \%$ e $1,59 \%$ de falso negativo.

A Tabela 3 evidencia os resultados com o classificador MLP, onde percebe-se uma taxa média de acerto de 95,42\%. Comparando os resultados do SVM com o MLP, percebe-se que a taxa média de acerto do SVM obteve um ganho de 4,02\%. Já para a especificidade a SVM atingiu $98,41 \%$ para $92,68 \%$ da MLP, representando um ganho de $5,73 \%$. Para a taxa de falso positivo constatou-se que a MLP obteve 5,65\% para $1,59 \%$ da SVM.

Tabela 3. Resultados dos Testes com MLP

\begin{tabular}{llcl}
\hline Folds & Taxa de Acerto (\%) & Taxa de erro (\%) & Situação \\
\hline 1 & 97,28 & 2,72 & \\
2 & 96,56 & 3,44 & \\
3 & 90,72 & 9,28 & Pior Caso \\
4 & 99,29 & 0,71 & \\
5 & 93,37 & 6,63 & \\
6 & 94,43 & 5,57 & \\
7 & 93,62 & 6,38 & \\
8 & 98,29 & 1,71 & \\
9 & 99,49 & 0,51 & Melhor Caso \\
10 & 91,21 & 8,79 & \\
\hline- & 95,42 & 4,58 & Caso Médio \\
\hline
\end{tabular}




\section{Conclusões e Trabalhos Futuros}

O presente estudo relatou a utilização dos classificadores SVM e MLP como ferramentas de auxílio na detecção de traços autísticos em adultos, com base em um banco de dados composto por 1.210 amostras de adultos com/sem autismo. Os resultados se mostraram satisfatórios de modo que o classificador SVM obteve os melhores resultado comparados aos obtidos pela MLP com taxas media de $99,48 \%$ e $95,42 \%$ respectivamente. Quando comparados os resultados pode-se inferir que o classificador SVM obteve ganhos na especificidade e com baixa taxa de falso positivo de $1,59 \%$ para $5,65 \%$ da MLP.

Como trabalhos futuros sugere-se a utilização de deep learning a fim de comparar os resultados obtidos levando em consideração as taxas médias de acertos (acurácia), a sensibilidade e especificidade.

\section{Referências}

Alves, A. L. C. et al. (2019). Funcionalidade em adultos com transtorno do espectro autista: relações entre traços autísticos, variáveis psicossociais e funcionamento cognitivo.

Alves, R. H. F., de Deus Júnior, G. A., Vieira, F. H. T., de Castro, M. S., de Araújo, S. G., and Lemos, R. P. (2016). Detecção de anomalias em painéis fotovoltaicos utilizando redes abnet, psom e mlp.

Araujo, M. D. A., Moreira, L. Y. M. R., and de Brito, R. X. (2019). Modelo computacional com fuzzy como recurso auxiliador na predição da esquizofrenia em adultos. In Anais da VII Escola Regional de Computação Aplicada à Saúde, pages 199-204. SBC.

Bentes, C. C. A., Barbosa, D. C., Fonseca, J. R. M., and Bezerra, L. C. (2016). A família no processo de inclusão social da criança e adolescente com autismo: Desafios na sociedade contemporânea. Intertem@s Social ISSN 1983-4470, 11(11).

Borges, M. and Shinohara, H. (2007). Síndrome de asperger em paciente adulto: Um estudo de caso. Revista Brasileira de Terapias Cognitivas, 3(1):41-48.

Caminha, V. L., Huguenin, J. Y., Assis, L. M. d., and Alves, P. P. (2016). Autismo: vivências e caminhos. São Paulo: Blucher, 11.

Carvalho, S. P., Lima, A. M., Brentani, H. P., Brunoni, D., Fock, R. A., and Nunes, F. L. S. (2016). Uma contribuição ao auxíllio do diagnóstico do autismo a partir do processamento de imagens para extração de medidas antropométricas. Revista de Informática Teórica e Aplicada, 23(2):100-123.

da Silva Talarico, M. V. T., dos Santos Pereira, A. C., and de Noronha Goyos, A. C. (2019). A inclusão no mercado de trabalho de adultos com transtorno do espectro do autismo: uma revisão bibliográfica. Revista Educação Especial, 32:119-1.

de Brito, R. X., Fernandes, C. A. R., and Amora, M. A. B. (2019). Análise de desempenho com redes neurais artificiais, arquiteturas mlp e rbf para um problema de classificação de crianças com autismo. iSys-Revista Brasileira de Sistemas de Informação, 13(1):60-76.

Freire Monteiro, A., de Almeida Pimenta, R., Pereira, S. M., and Roesler, H. (2018). Considerações sobre critérios diagnósticos de transtorno do espectro autista, e suas implicações no campo científico. DO CORPO: ciências e artes, 7(1). 
Haykin, S. S. et al. (2009). Neural networks and learning machines/simon haykin.

Lorena, A. C. and de Carvalho, A. C. (2007). Uma introdução às support vector machines. Revista de Informática Teórica e Aplicada, 14(2):43-67.

Martins, C. M. L. (2017). Musicoterapia e as competências sociais do adulto com deficiência. Master's thesis.

Onzi, F. Z. and de Figueiredo Gomes, R. (2015). Transtorno do espectro autista: a importância do diagnóstico e reabilitação. Revista Caderno Pedagógico, 12(3).

Pinheiro, T. D. (2018). Classificação de imagens faciais para o auxílio ao diagnóstico do transtorno do espectro autista. PhD thesis, Universidade de São Paulo.

Santos, E. J. R. d. (2019). Previsão de precipitação usando máquinas de vetores de suporte visando sua implementação em sistemas embarcados.

Santos, E. M. d. et al. (2002). Teoria e aplicação de support vector machines à aprendizagem e reconhecimento de objetos baseado na aparência.

Semolini, R. et al. (2002). Support vector machines, inferência transdutiva e o problema de classificação.

Souza, B., Teixeira, A., and Silva, d. A. (2009). Classificação de bioma caatinga usando support vector machines (svm). Anais XIV Simpósio Brasileiro de Sensoriamento Remoto, pages 7917-7924.

Souza, L. L. d. (2016). Análise da pressão plantar da marcha de autistas por dinâmica simbólica otimizada por algoritmo genético.

Zavaleta, J., Costa, M., Cruz, S., Manhaes, L., Carvalho, L., and Mousinho, R. (2012). Dysdtool: Uma ferramenta inteligente para a avaliação e intervenção no apoio ao diagnóstico da dislexia. In CSBC-XII Workshop de Informática Médica.

Zilbovicius, M., Meresse, I., and Boddaert, N. (2006). Autismo: neuroimagem. Brazilian Journal of Psychiatry, 28:s21-s28. 